\section{Imaging vitreous}

J Sebag

\begin{abstract}
Purpose Imaging vitreous is a quest to view what is, by design, invisible. This treatise will review significant historical aspects, past and present imaging methodologies, and imaging techniques that are currently in development for future research and clinical
\end{abstract} applications.

Methods Classic and modern histologic techniques, dark-field slit microscopy, clinical slit lamp biomicroscopy, standard and scanning laser ophthalmoscopy, ultrasonography, optical coherence tomography, magnetic resonance and raman spectroscopy, and dynamic light scattering methodologies are presented.

Results The best available histologic techniques for imaging vitreous are those that avoid rapid dehydration of vitreous specimens. Dark-field slit microscopy enables in vitro imaging without dehydration or tissue fixatives. Optical coherence tomography enables better in vivo visualization of the vitreo-retinal interface than scanning laser ophthalmoscopy and ultrasonography but does not image the vitreous body. Dynamic light scattering can determine the average sizes of vitreous macromolecules within the vitreous body as well as possibly image the posterior vitreous cortex once detached, while Raman spectroscopy can detect altered vitreous molecules, such as glycated collagen and other proteins in diabetic vitreopathy. Conclusions The inability to adequately image vitreous hinders a more complete understanding of its normal structure and how this changes in aging and disease. The combined use of more than one technique could provide better imaging of vitreous for future investigational and clinical purposes. Eye (2002) 16, 429-439. doi:10.1038/ sj.eye. 6700201

Keywords: vitreous; imaging; biochemistry; vitreoretinal interface; posterior vitreous detachment; diabetic vitreopathy; vitreoschisis

\section{Introduction}

Duke-Elder $^{1}$ once described that the first theories of vitreous structure proposed that vitreous is composed of 'loose and delicate filaments surrounded by fluid', a description that is remarkably close to present day concepts. During the 18th and 19th centuries there were no less than four very different theories of vitreous structure. In 1741 Demours formulated the Alveolar Theory, claiming that there are alveoli of fluid between fibrillar structures. In 1780, Zinn proposed that vitreous is arranged in a concentric, lamellar configuration similar to the layers of an onion. The dissections and histologic preparations of Von Pappenheim and Brucke provided evidence for this Lamellar Theory. The Radial Sector Theory was proposed by Hannover in 1845. Studying coronal sections at the equator, he described a multitude of sectors approximately radially oriented around the central anteroposterior core that contains Cloquet's canal. Hannover likened this structure to the appearance of a 'cut orange'. In 1848 Sir William Bowman established the Fibrillar Theory, which was based upon his finding microscopic fibrils, an observation which confirmed Retzius's earlier description of fibers that arose in the peripheral anterior vitreous and assumed an undulating pattern in the central vitreous, similar to a 'horse's tail'. In 1917 the elegant histologic preparations of Szent-Györgi supported these observations and introduced the concept that vitreous structure changes with age.

Unfortunately, the techniques employed in all these studies were flawed by artifacts that biased the results of the investigations. As pointed out by Baurmann and Redslob, ${ }^{2}$ these early histologic studies employed acid tissue fixatives that precipitated what we recognize today as the glycosaminoglycans, hyaluronan, formerly called hyaluronic acid, an effect which altered the histologic imaging of vitreous. Thus, the development of slit lamp biomicroscopy by Gullstrand in 1912 held great promise, as it was anticipated that this technique could enable imaging of vitreous
Associate Clinical Professor of Ophthalmology Doheny Eye Institute Keck School of Medicine University of Southern California CA, USA

Correspondence: J Sebag, MD 18821 Delaware Street Huntington Beach CA 92648, USA

Tel: 714-841-5002

Fax: 714-843-5620 E-mail: jsebag@pol.net

The author has no propriety interest in the imaging technologies described herein 
structure without the introduction of fixation artifacts. Yet, as described by Redslob ${ }^{2}$ a varied set of descriptions resulted over the years, ranging from a fibrous structure to sheets, 'chain-linked fences', and various other interpretations. This problem even persisted in more recent investigations. Eisner ${ }^{3}$ described 'membranelles', Worst ${ }^{4}$ 'cisterns', Sebag and Balazs $^{5}$ 'fibers', and Kishi and Shimizu 'pockets' in the vitreous. The observation of these so-called 'pockets' by the last-mentioned group was ultimately found to be an age-related phenomenon with little relevance to the normal macromolecular structure. ${ }^{7}$

Previously considered a vestigial organ, vitreous is now regarded as an important ocular structure, ${ }^{8,9}$ at least with respect to several important pathologic conditions of the posterior segment. This remarkable tissue is in essence an extended extracellular matrix, composed largely of water with a very small amount of structural macromolecules. ${ }^{9,10}$ Nevertheless, in the normal state it is a solid and clear gel, especially in youth (Figure 1). Because of the predominance of water within vitreous, effective imaging of this structure in vitro is best performed by methods that overcome the intended transparency of this tissue yet avoid dehydration. Imaging vitreous in vivo is likely best achieved by visualizing the macroscopic features via an assessment of the nature and organization of the molecular components. The following will review some of the most important methods available for imaging vitreous in vitro and in vivo.

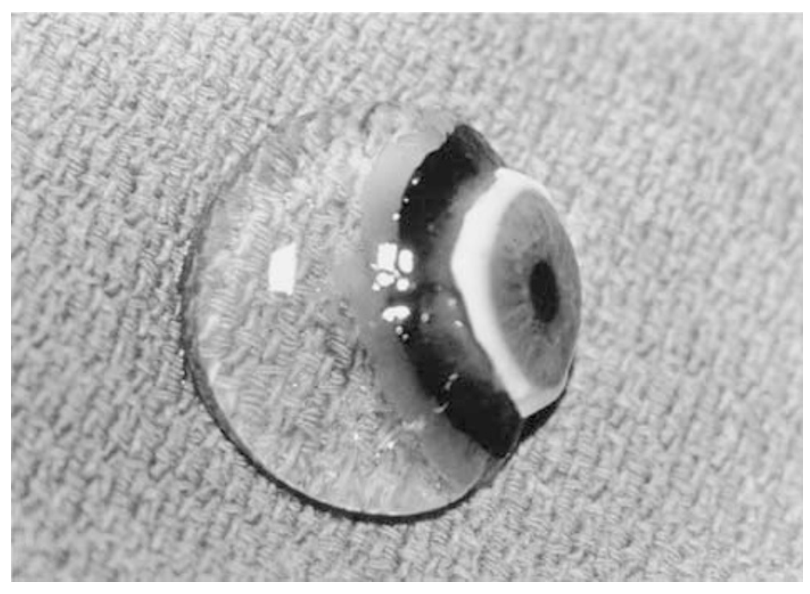

Figure 1 Human vitreous body. Human vitreous of a 9-monthold child dissected of the sclera, choroid, and retina, is still attached to the anterior segment. Although the specimen is situated on a surgical towel in room air, the vitreous maintains its shape, because in youth the vitreous body is nearly entirely gel (specimen courtesy of the New England Eye Bank).

\section{Methodologies}

\section{In vitro imaging}

Arguably the best available technique for histologic characterization of vitreous structure is currently in use by Faulborn. Through an arduous process of tissue preparation that very slowly dehydrates specimens over months, this technique minimizes the disruption of vitreous structure that results from the rapid dehydration that is induced by standard histologic tissue processing. The elegant preparations obtained with such slow dehydration have provided great insight into the role of vitreous in the pathophysiology of proliferative diabetic vitreoretinopathy ${ }^{11}$ (Figure 2) and retinal tears ${ }^{12}$ (Figure 3).

Dark-field slit microscopy of whole human vitreous in the fresh, unfixed state was extensively employed by Sebag and Balazs to characterize the fibrous structure of vitreous ${ }^{13}$ (Figure 4), age-related changes within the central vitreous body ${ }^{14}$ and at the vitreoretinal interface, ${ }^{15}$ and the effects of diabetes on human vitreous structure. ${ }^{16}$ This imaging method has clearly demonstrated the fibers in the anterior peripheral vitreous (Figure 5) that transmit traction to the peripheral retina in rhegmatogenous retinal pathology. Fibers in this region also play a role in the formation of the so-called 'anterior loop' configuration of anterior proliferative vitreoretinopathy (Figure 6).

\section{In vivo imaging}

Ophthalmoscopy and biomicroscopy Of all the parts of the eye that are routinely evaluated by clinical inspection, vitreous is perhaps the least amenable to standard examination techniques. This is because examining vitreous is an attempt to visualize a structure designed to be virtually invisible. ${ }^{17}$ With the Direct Ophthalmoscope light rays emanating from a point in the patient's fundus emerge as a parallel beam which is focused on the observer's retina and an image is formed. However, incident light reaches only the part of the fundus onto which the image of the light source falls and only light from the fundus area onto which the observer's pupil is imaged reaches that pupil. Thus, the fundus can be seen only where the observed and the illuminated areas overlap and where the light source and the observer's pupil are aligned optically. This restricts the extent of the examined area and also because of a limited depth of field, this method is rarely used to assess vitreous structure.

Indirect Ophthalmoscopy extends the field of view by using an intermediate lens to gather rays of light from a wider area of the fundus. Although binocularity 


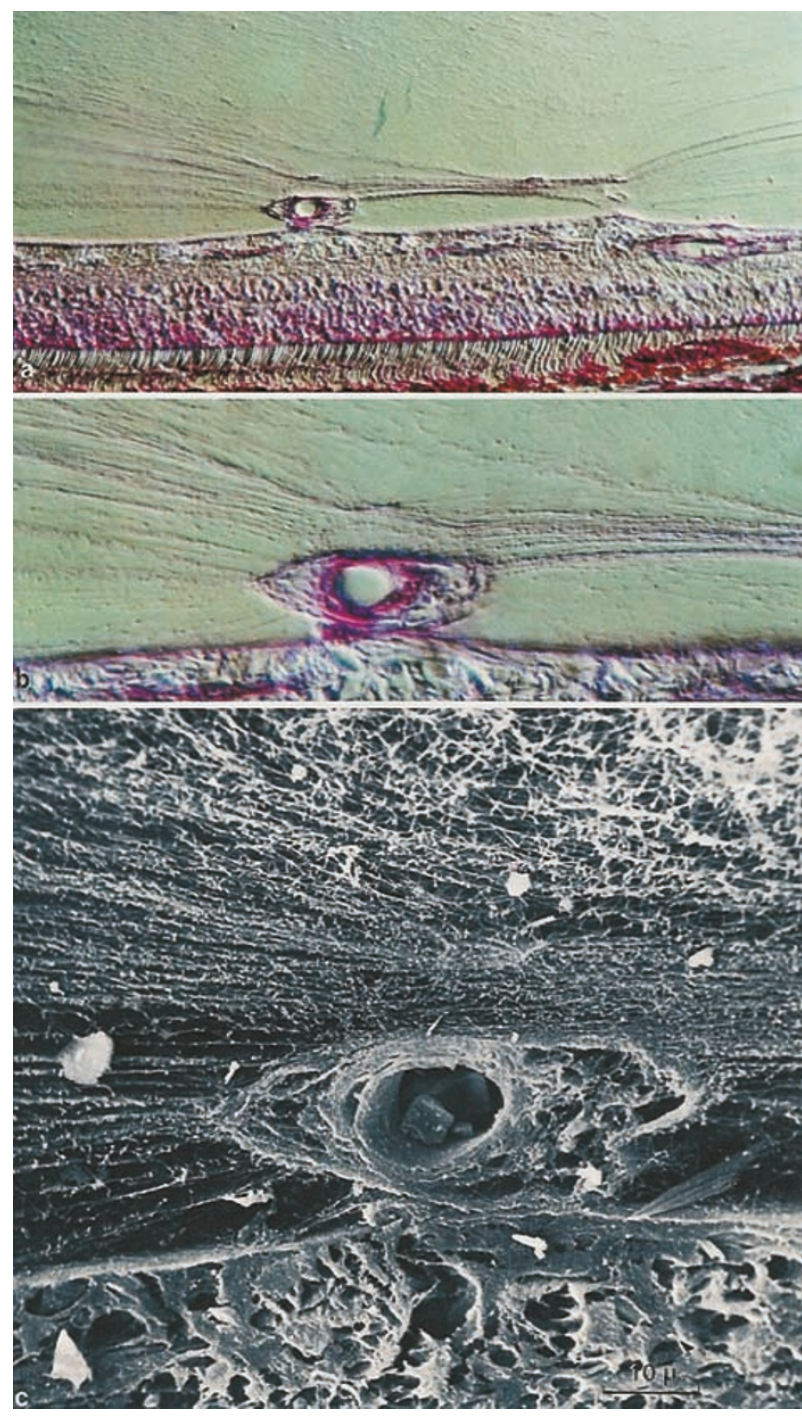

Figure 2 Proliferative diabetic vitreo-retinopathy. Neovascularization which arises from the disc and retina involves vascular endothelial cell migration and proliferation onto and into the posterior vitreous cortex. These photomicrographs demonstrate the formation of neovascular complexes sprouting into the posterior vitreous cortex of a human eye (bar $=10 \mu \mathrm{m})$ (reprinted with permission ${ }^{11}$ ).

provides stereopsis, the image size is considerably smaller than with direct ophthalmoscopy and only significant alterations in vitreous structure, such as the hole in the prepapillary posterior vitreous cortex (Figure 7), vitreous hemorrhage, or asteroid hyalosis, are reliably diagnosed by indirect ophthalmoscopy.

Effectively using Slit Lamp Biomicroscopy to overcome vitreous transparency necessitates maximizing the Tyndall effect. Although this can be achieved in vitro, as described above, there are limitations to the illumination/observation angle that can be achieved clinically. This is even more troublesome in the presence of meiosis, corneal and/or lenticular opacities, and limited patient cooperation. Essential to the success of achieving an adequate Tyndall effect are maximizing pupil dilation in the patient, since the Tyndall effect increases with an increasingly subtended angle between the plane of illumination and the line of observation (up to a maximum of $90^{\circ}$ ), and dark adaptation in the examiner. Some observers purport that green light enhances the Tyndall effect, although this has never been explained or tested scientifically. Preset Lens Biomicroscopy attempts to increase the available illumination-observation angle, offers dynamic inspection of vitreous in vivo, and provides the capability of recording the findings in real-time. ${ }^{18}$ Initially introduced on a wide-scale for use with a Hruby lens and currently practised by using a handheld 90 diopter lens at the slit lamp, this technique is purportedly best performed with a fundus camera and the El Bayadi-Kajiura lens promoted by Schepens, Trempe, and associates. This approach has been used in many seminal studies of the role of vitreous in various disease states. ${ }^{18}$ However, there has not been widespread use of this approach, probably because it is heavily dependent upon subjective interpretation of the findings and questionable reproducibility from center to center.

The Scanning Laser Ophthalmoscope (SLO), also developed at the Schepens Eye Research Institute in Boston, enables dynamic inspection of vitreous in vivo, features tremendous depth-of-field, and offers real-time recording of findings. Monochromatic green, as well as other wavelengths of light are available for illumination. ${ }^{19}$ To date, however, the SLO has really only improved our ability to visualize details in the prepapillary posterior vitreous, such as Weiss's ring (Figure 8). Unfortunately, in spite of the dramatic depth-of-field possible with this technique, SLO does not adequately image the vitreous body and an attached posterior vitreous cortex, probably because of limitations in the level of resolution. Thus, posterior vitreous detachment (PVD), by far the most common diagnosis to be entertained when imaging vitreous clinically, is not reliably determined by SLO. Indeed, there is an increasing awareness among vitreous surgeons that the reliability of the clinical diagnosis of total posterior vitreous detachment by any existing technique is woefully inadequate. This awareness arises from the fact that vitreous surgery following clinical examination often reveals findings that are contradictory to preoperative assessments.

Ultrasonography Ultrasound is an inaudible acoustic wave that has a frequency of more than $20 \mathrm{kHz}$. The frequencies used in ophthalmology are generally in the 
a

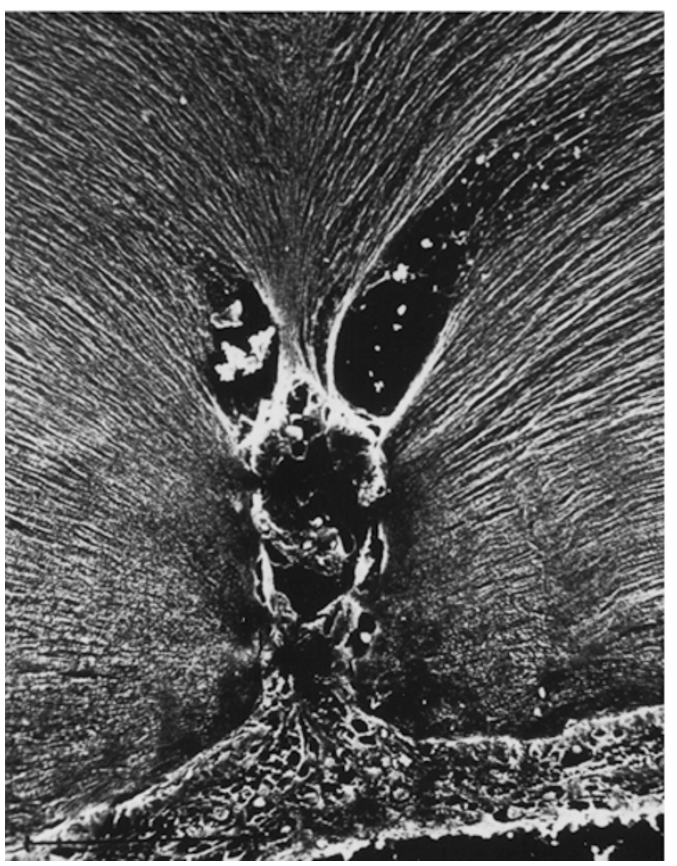

b

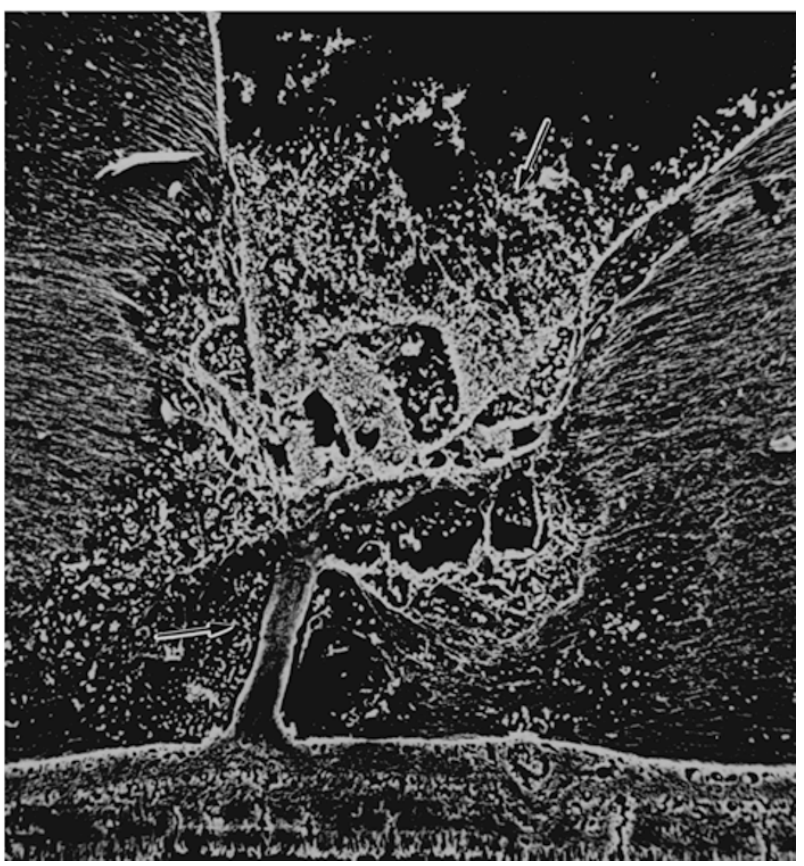

Figure 3 Morphology of peripheral vitreous. (a) Cystic retinal tuft. The tuft is a cystoid formation of fibers, similar to those of the nerve fiber layer, and cells, similar to those found in the inner plexiform layer of the retina. The tuft is connected to the internal limiting lamina of the retina. This scanning electron micrograph shows the insertion of the vitreous collagen fibers on the tuft's apical surface. Their orientation changes toward the tuft's surface (reprinted with permission ${ }^{12}$ ). (b) Verruca. The verruca has a structure similar to that of a tree. Its 'roots' are embedded in the inner layers of the retina. Cellular elements resembling cells of the inner plexiform layer can be seen near the retinal surface. The 'trunk' of this structure extends from the retina to the middle parts of the vitreous cortex. The 'branches' of the verruca are intertwined with interrupted vitreous collagen fibers. Local condensation of collagen fibers exists as well as local collagen destruction (arrows) and interruption of the internal limiting lamina of the retina (reprinted with permission ${ }^{12}$ ).

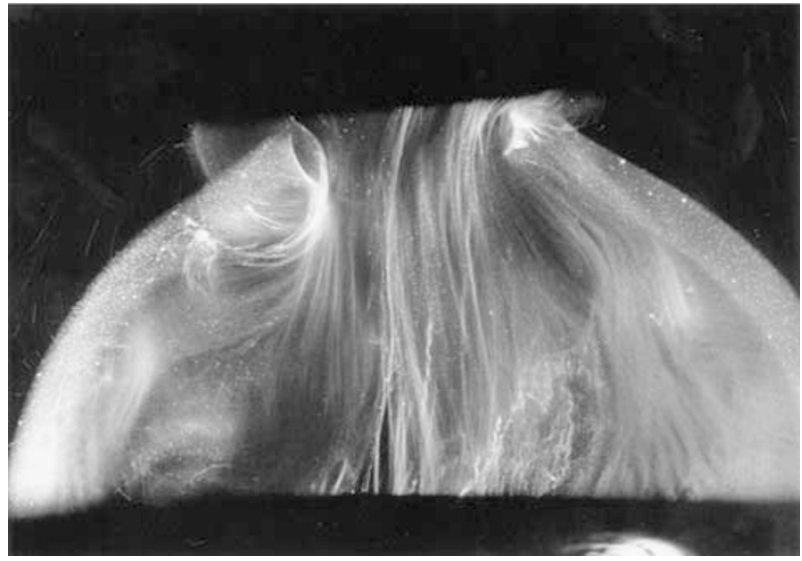

Figure 4 Posterior and central vitreous of a 59-year-old male. The premacular hole is to the top at the center. Fibers course anteroposteriorly in the center of the vitreous and enter the retrocortical (preretinal) space via the premacular region of the vitreous cortex. Within the cortex are many small 'dots' that scatter light intensely. The larger, irregular dots are debris. The small dots are hyalocytes.

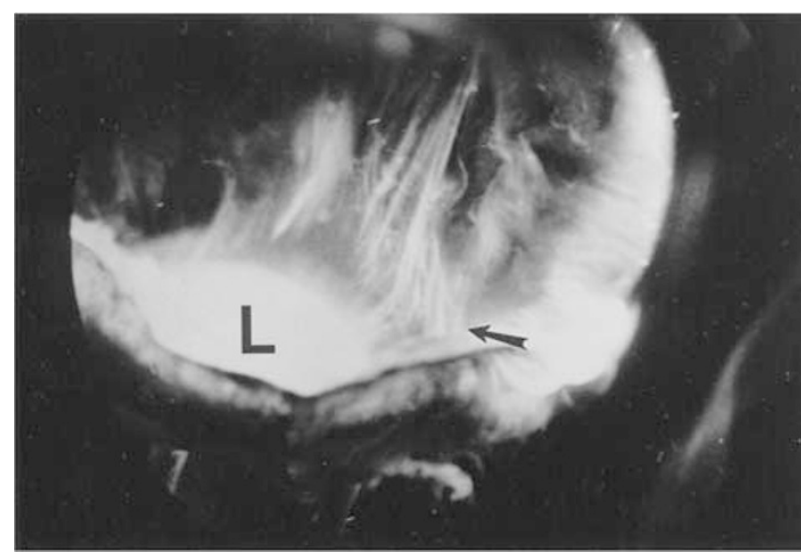

Figure 5 Vitreous base morphology. Vitreous structure in a 58year-old female. Fibers course anteroposteriorly in the central and peripheral vitreous. Posteriorly, fibers orient to the premacular region. Anteriorly, the fibers 'splay out' to insert into the vitreous base (arrow). $\mathrm{L}=$ crystalline lens. 


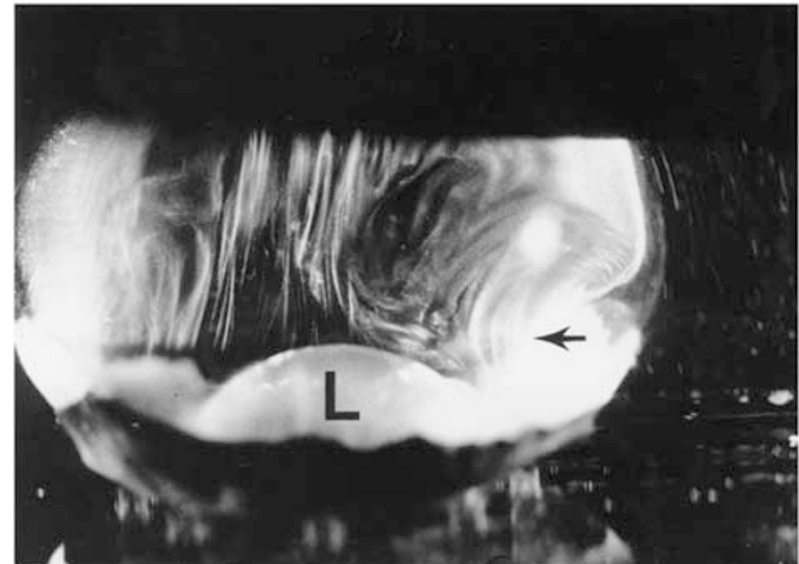

Figure 6 Vitreous base 'anterior loop'. Central and peripheral vitreous structure in a 76-year-old male. The posterior aspect of the lens is seen below. Fibers course anteroposteriorly in the central vitreous and insert at the vitreous base. The 'anterior loop' configuration at the vitreous base is seen on the right side of the specimen. $\mathrm{L}=$ lens, arrow $=$ anterior loop of vitreous base. Reprinted with permission from Sebag J, Balazs EA. Pathogenesis of cystoid macular edema: an anatomic consideration of vitreoretinal adhesions. Surv Ophthalmol 1984; 28 (suppl): 493498.

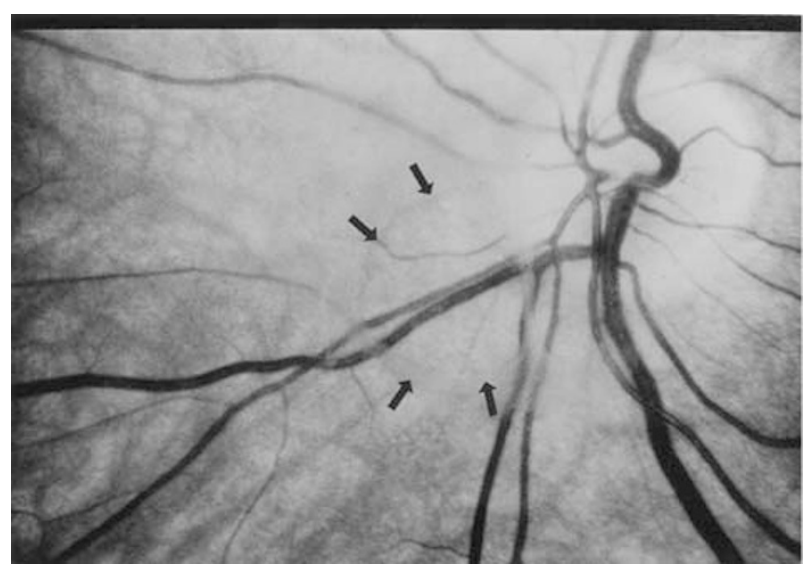

Figure 7 Fundus photograph of prepapillary PVD. The posterior vitreous is detached and the prepapillary hole in the posterior vitreous cortex (arrows) can be seen detached anterior to the optic disc (slightly below and to the left of the optic disc in this photograph of the left eye).

range 8-10 MHz. Although these very high frequencies produce wavelengths as short as $0.2 \mathrm{~mm}$, these are not short enough to adequately assess normal internal vitreous structure such as the fibers described above. Even the posterior vitreous cortex, about $100 \mu \mathrm{m}$ at its thickest point in the normal state, is below the level of resolution of conventional ultrasonography. The utility of this technique results from the fact that strong echoes are produced at 'acoustic' interfaces found at the junctions of media with different densities and
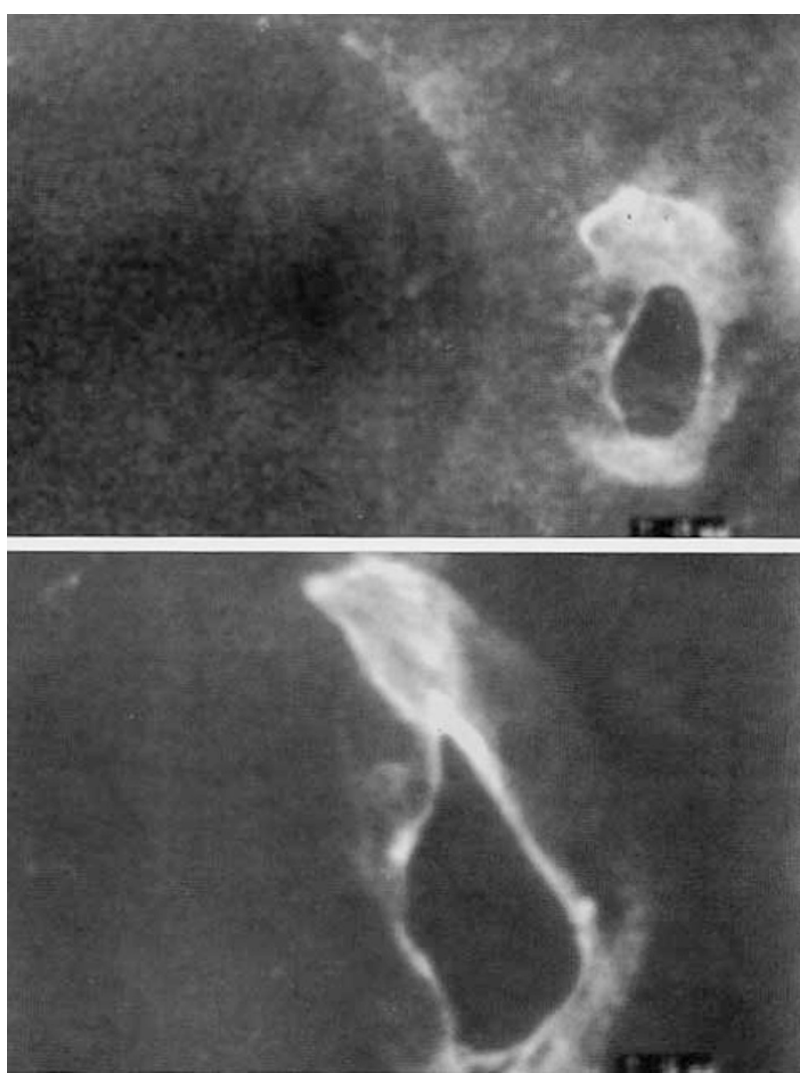

Figure 8 Scanning laser ophthalmoscopy (SLO) of Weiss's ring. SLO image of detached posterior vitreous cortex demonstrates the appearance of Weiss's ring surrounding the prepapillary hole (reprinted with permission ${ }^{18}$ ).

sound velocities. The greater the difference in density between the two media that create the acoustic interface, the more prominent the echo. Thus, agerelated or pathologic phase alterations within the vitreous body are detectable by ultrasonography.

Oksala, among the first to employ B-scan ultrasonography to image vitreous in the late 1950s and early 1960s, summarized his findings of aging changes in $1978 .^{20}$ In that report of 444 'normal' subjects, Oksala defined the presence of acoustic interfaces within the vitreous body as evidence of vitreous aging and determined that the incidence of such interfaces was 5\% between the ages of 21-40 years, and fully $80 \%$ of individuals over 60 years of age. In clinical practice, however, only profound entities such as asteroid hyalosis, vitreous hemorrhage, and intravitreal foreign bodies (if sufficiently large) are imaged by ultrasonography. At the vitreo-retinal interface, the presence of a posterior vitreous detachment is often suspected on the basis of B-scan ultrasonography but can never be definitively established, since the level of resolution is not sufficient to reliably image the posterior vitreous 
cortex, only a little more than $100 \mu \mathrm{m}$ thick at its thickest portion. However, recent studies have successfully used this technique to determine the presence of a split in the posterior vitreous cortex, called vitreoschisis (Figure 9), of patients with proliferative diabetic vitreo-retinopathy. ${ }^{21}$ The success achieved in using ultrasound to identify this important
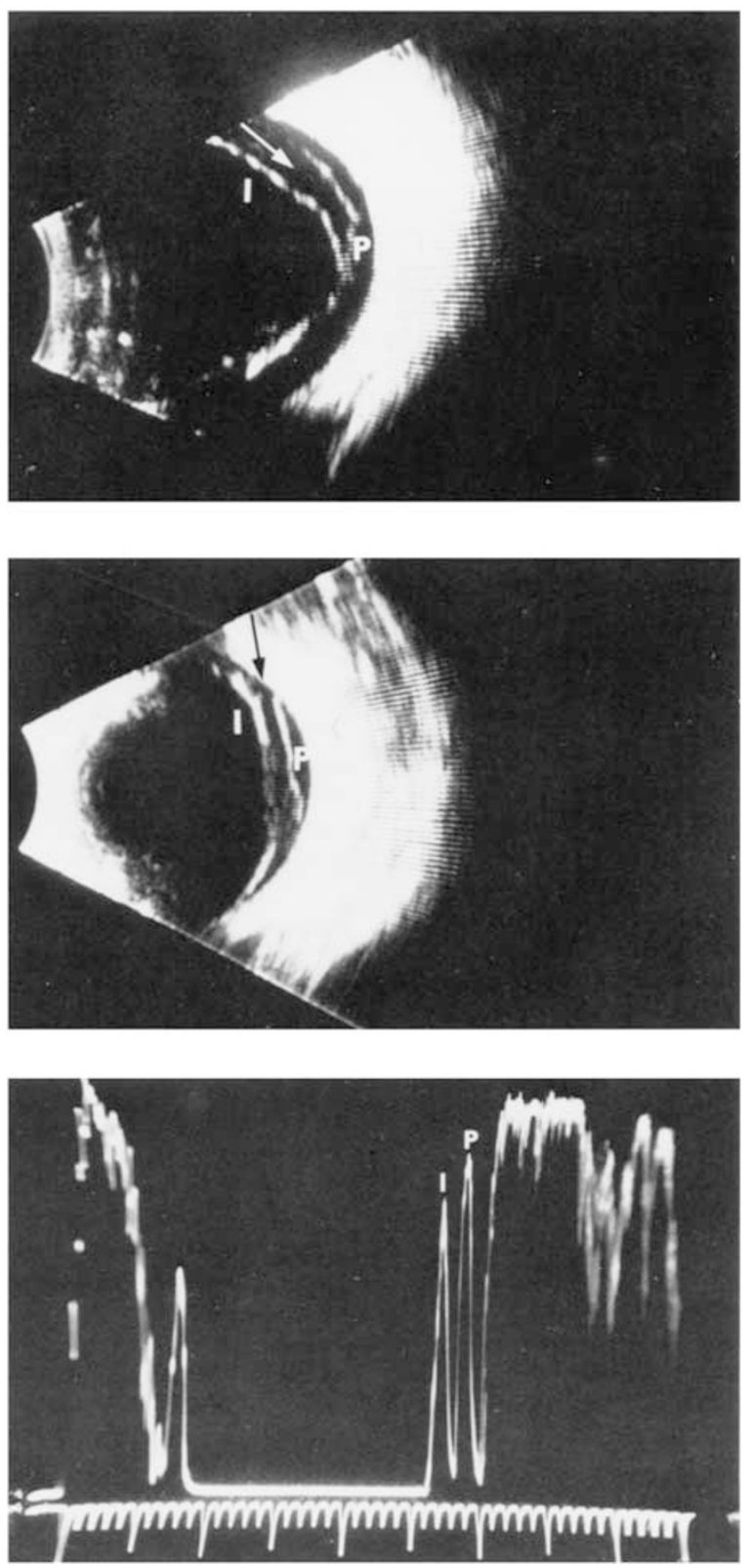

Figure 9 Ultrasonography imaging of vitreoschisis. Splitting of the vitreous cortex (arrow) can occur and mimic posterior vitreous detachment. In diabetic patients, blood can be present in the vitreoschisis cavity. ( $\mathrm{I}=$ inner wall, $\mathrm{P}=$ posterior wall of vitreoschisis cavity within the posterior vitreous cortex.) Photograph courtesy of Dr Ronald Green. Reprinted with permission from Green RL, Byrne SF. Diagnostic ophthalmic ultrasound. In: Ryan SJ (ed). Retina. Mosby: St Louis, 1989. pathologic entity probably results from the fact that this tissue is abnormally thickened by non-enzymatic glycation of vitreous collagen and other proteins. ${ }^{22}$ In the absence of such advanced disease, however, the diagnosis of PVD cannot be reliably established by ultrasonography.

Optical coherence tomography (OCT) Introduced in 1991, optical coherence tomography (OCT) is a new

technique for high-resolution cross-sectional imaging of ocular structure. ${ }^{23}$ OCT is based on the principle of low coherence interferometry, where the distances between and sizes of structures in the eye are determined by measuring the 'echo' time it takes for light to be backscattered from the different structures at various axial distances. The resolution of all echo-based instrumentation (such as ultrasound and OCT) is based upon the ratio of the speed of the incident wave to that of the reflected wave. As described above, clinical ultrasonography is performed with a frequency of approximately $10 \mathrm{~Hz}$ and has a $150 \mathrm{~mm}$ resolution. Although recently introduced ultrasound biomicroscopy has increased the frequency (up to $100 \mathrm{~Hz}$ ), and thus has a spatial resolution of $20 \mu \mathrm{m}$, penetration into the eye is no more than $4-5 \mathrm{~mm}$. Light-based devices, such as the OCT, use an incident wavelength of $800 \mathrm{~nm}$ and have increased axial resolution to $10 \mu \mathrm{m}$, providing excellent imaging of retinal architecture, although vitreous applications are less useful. The limitations of OCT include the inability to obtain high-quality images through media opacities such as dense cataract or vitreous hemorrhage. The use of OCT is also limited to cooperative patients who are able to maintain fixation for the full acquisition time of 2.5 seconds per section. Thus, to date OCT has primarily been used to image, and to some extent quantitate, retinal laminar structure. Some vitreous applications have been useful, especially those that involve imaging the vitreo-macular interface in patients with macular holes ${ }^{24}$ (Figure 10). However the exact nature and molecular composition of these pre-retinal tissue planes cannot be definitively deduced using OCT and little information can be garnered about structures within the vitreous body.

\section{Spectroscopy}

Nuclear magnetic resonance (NMR) spectroscopy The NMR phenomenon is based upon the fact that when placed in a magnetic field magnetic nuclei, such as water protons, tend to orient their magnetic vectors along the direction of the magnetic field. The time constant for this orientation, known as the longitudinal relaxation time $T_{1}$, reflects the thermal interactions of 


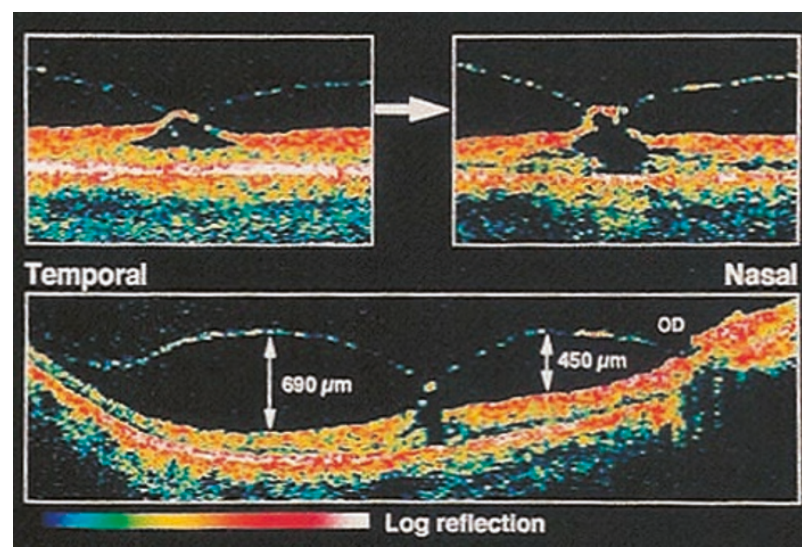

Figure 10 OCT of macular hole. Evolution of an impending macular hole in a fellow eye to a stage 2 hole. Top left, OCT shows a cystic formation in the inner part of the foveal center. The elevation of the foveal floor and the adherence of the partially detached posterior vitreous cortex suggest vitreoretinal traction. Top right, 2 months later, the roof of the cyst has opened on the nasal side of the macula, constituting an incompletely detached operculum to which the posterior vitreous cortex adheres. Bottom, Composite optical coherence tomogram shows the convex elevation of the partially detached posterior vitreous cortex, suggesting anteroposterior vitreous traction (reprinted with permission ${ }^{24}$ ).

protons with their molecular environment. Magnetic vectors that have previously been induced to be in phase with each other undergo a 'dephasing' relaxation process that is measured by the transverse relaxation time $T_{2}$. It is the transverse relaxation time $T_{2}$ that reflects inhomogeneities within the population of protons. Protons oriented by a magnetic field absorb radio waves of the appropriate frequency to induce transactions between their two orientations. Such absorption is the basis of the NMR signal used to index relaxation times. Relaxation times in biologic tissues vary with the concentration and mobility of water within the tissue. As the latter is influenced by the interaction of water molecules with macromolecules in the tissue, this non-invasive measure can assess the gel to liquid transformation that occurs in vitreous during aging ${ }^{14}$ and disease states, such as diabetic vitreopathy. ${ }^{16,25}$ These considerations led Aguayo et $a l^{26}$ to use NMR in studying the effects of pharmacologic vitreolysis ${ }^{27}$ of bovine and human vitreous specimens and intact bovine eyes in vitro. Collagenase induced measurable vitreous liquefaction more so than hyaluronidase (Figure 11). Thus, this non-invasive method could be used to evaluate age and disease-induced synchisis (liquefaction) of the vitreous body, although it is not clear that this technique would adequately evaluate the vitreo-retinal interface. There have, curiously, been few recent studies that have employed NMR spectroscopy in research or clinical applications on vitreous.

Raman spectroscopy This form of spectroscopy was first described in 1928 by CV Raman in India. Raman spectroscopy is an inelastic light scattering technique wherein the vibrational mode of molecules in the study specimen absorb energy from incident photons, causing a downward frequency shift, which is called the Raman shift. Because the signal is relatively weak, current techniques employ laser-induced stimulation with gradual increases in the wavelength of the stimulating laser, so as to be able to detect the points at which the Raman signal becomes apparent as peaks superimposed on the broad background fluorescence. The wavelengths at which these peaks are elicited are characteristic of the chemical bonds, such as aliphatic $\mathrm{C}-\mathrm{H}\left(2939 \mathrm{~cm}^{-1}\right)$, water O-H $\left(3350 \mathrm{~cm}^{-1}\right), \mathrm{C}=\mathrm{C}$ and C$\mathrm{H}$ stretching vibrations in $\pi$-conjugated and aromatic molecules $\left(1604 \mathrm{~cm}^{-1}\right.$ and $\left.3057 \mathrm{~cm}^{-1}\right)$, etc. To date, most applications of this technique in the eye have been for analysis of lens structure and pathology. ${ }^{28}$ The use of near-infrared (IR) excitation wavelengths is particularly effective in the lens, since these wavelengths have better penetration in opacified cataractous lenses.

Studies ${ }^{29}$ in vitreous employed excised samples of human vitreous obtained during surgery. The near-IR excitation at $1064 \mathrm{~nm}$ was provided by a diodepumped Nd:YAG CW laser with a diameter of $0.1 \mathrm{~mm}$ and a power setting of $300 \mathrm{~mW}$. Back-scattering geometry with an optical lens collected scattered light which was passed through a Rayleigh light rejection filter into a spectrophotometer. The results (Figure 12) showed that this technique was able to detect peaks at $1604 \mathrm{~cm}^{-1}$ and $3057 \mathrm{~cm}^{-1}$ in vitreous of diabetic patients that were not present in controls. Further research and development is needed to reliably interpret such results and refine the methodology for non-invasive use in situ. While this has already been achieved in the lens, it is not clear that this will be possible for vitreous applications.

Dynamic light scattering (DLS) DLS is an established laboratory technique to measure average size (or size distribution) of microscopic particles as small as $3 \mathrm{~nm}$ in diameter that are suspended in a fluid medium where they undergo random Brownian motion. Light scattered by a laser beam passing through such a dispersion will have intensity fluctuations in proportion to the Brownian motion of the particles. Since the size of the particles influences their Brownian motion, analysis of the scattered light intensity yields a distribution of the size(s) of the suspended particles. 

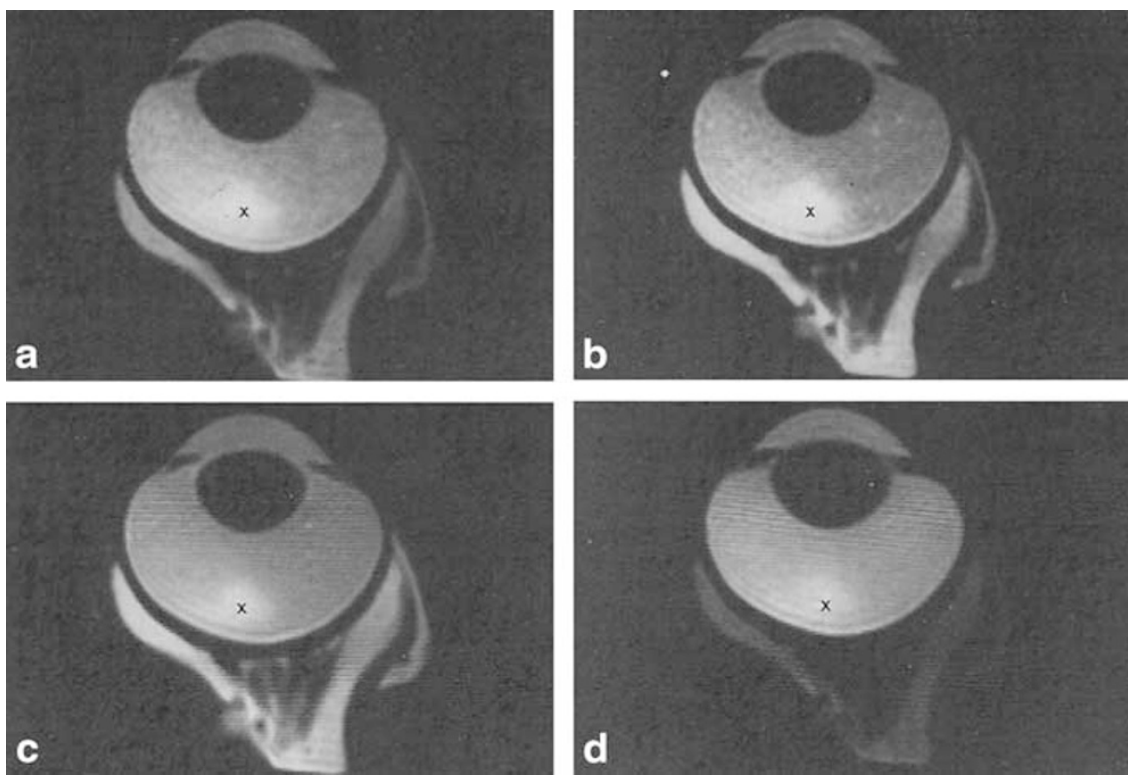

Figure 11 NMR spectroscopy of pharmacologic vitreolysis. Progressive saturation (a) (300 ms), (b) (1200 ms), (c) (2000 ms), (d) $(3920 \mathrm{~ms})$ image of a bovine eye $12 \mathrm{~h}$ after injection with $0.2 \mathrm{ml}$ of $100 \mathrm{U} / \mathrm{ml}$ collagenase solution. Note the bright area (x) in the posterior pole. This area of enhanced relaxation (shorter $\mathrm{T}_{1}$ ) was absent immediately after injection (reprinted with permission ${ }^{26}$ ).

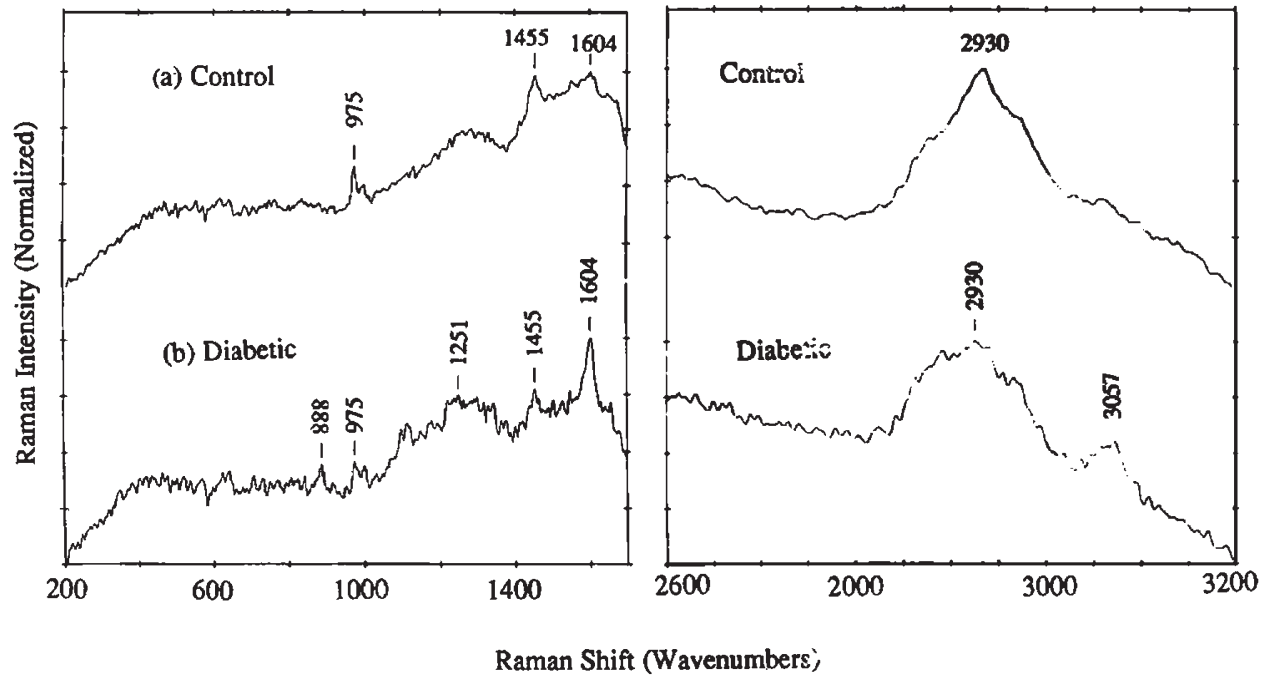

Figure 12 Raman spectroscopy of diabetic vitreopathy. Fourier Transform Raman spectra (using a laser power $=300 \mathrm{~mW}$, laser spot diameter $=0.1 \mathrm{~mm}$ and 250 coadded scans) of control and diabetic human vitreous collagen. Samples were pooled into one specimen for the patients with diabetes $(n=7)$ and one for the control group $(n=10)$. The graphs represent the actual FT-RS spectra of these two specimens. Quantitative analysis of the peak area at $1604 \mathrm{~cm}^{-1}$ showed a three-fold increase in samples from patients with diabetes compared to controls. The broad background was corrected for when drawing the baseline of the peak at $1455 \mathrm{~cm}^{-1}$. Similarly, there was a peak at $3057 \mathrm{~cm}^{-1}$ in diabetic vitreous not present in controls (reprinted with permission ${ }^{29}$ ).

Visible light from a laser diode (power $50 \mu \mathrm{w}$ ) is focused into a small scattering volume inside the specimen (excised lens or vitreous, autopsy or living eye). The detected signal is processed via a digital correlator to yield a time autocorrelation function (TCF). For dilute dispersions of spherical particles the slope of the TCF provides a quick and accurate determination of the particle's translational diffusion coefficient, which can be related to its size via a Stokes-Einstein equation, provided the viscosity of the suspending fluid, its temperature, and its refractive index are known. For the lens and vitreous, a viscosity of $\eta=0.8904$ centipoise, a refractive index of $n=$ 1.333 , and a temperature of $25^{\circ} \mathrm{C}$ for in vitro studies and $37^{\circ} \mathrm{C}$ for in vivo studies were used to determine macromolecule sizes. 


\section{3-D Scan of a Bovine Vitreous}
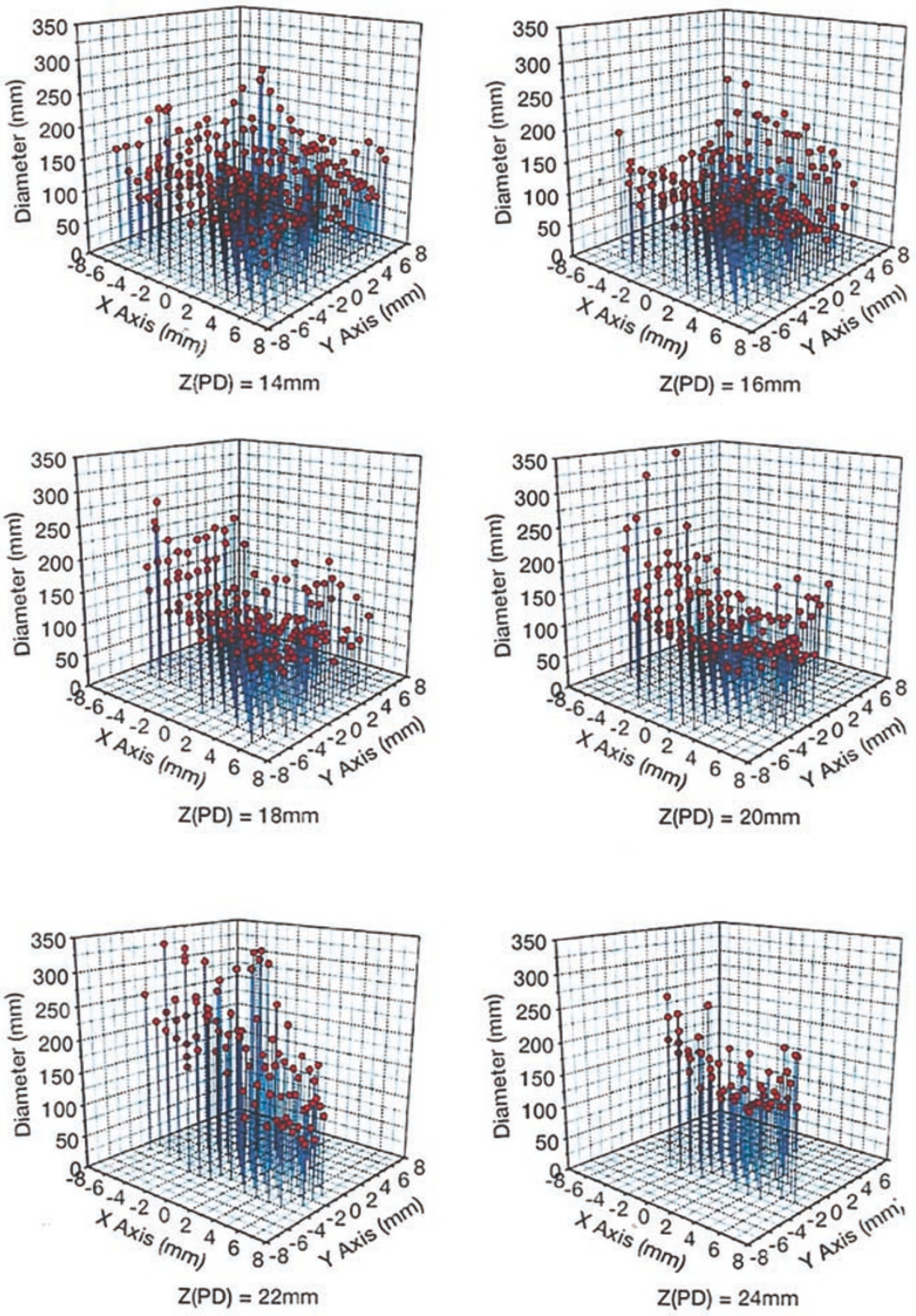

Figure 13 Dynamic light scattering (DLS) of bovine vitreous. Three-dimensional map of DLS measurements in the bovine vitreous body, in situ. The $x$-axis is the horizontal distance from a central (near-optical) axis of the eye. The $y$-axis is the distance along the sagittal (front-to-back) plane from the central starting point of measurements on the optical axis of the eye. From this starting point, whose location is represented as the $z$ coordinate and is defined as $\mathrm{mm}$ posterior to the lens along the optical axis in the central vitreous, measurements were obtained at $0.5 \mathrm{~mm}$ steps in both the $x$ and $y$ directions. Along the $z$-axis (in the sagittal plane), measurements were obtained every $2 \mathrm{~mm}$, beginning at $14 \mathrm{~mm}$ behind the back surface of the lens to $24 \mathrm{~mm}$ posterior to this point. The heterogeneous distribution of macromolecules throughout the vitreous body can be appreciated in these plots with the height of the lines representing the average particle size for all molecular constituents (cumulant fit of both fast hyaluronan and slow collagen) at the measurement site (reprinted with permission ${ }^{30}$ ). 
Studies $^{30-32}$ in the lens and vitreous employ DLS instrumentation that was developed at NASA to conduct fluid physics experiments on-board the space shuttle and space station orbiters. The input beam from a semi-conductor laser (670-nm wavelength) at $50 \mu \mathrm{W}$ power was projected into the specimens and the scattered signal was collected by the DLS probe for a duration of 10 seconds. The signal was then detected by an avalanche photodiode detector system. A TCF was constructed using a digital correlator card. The slope of the TCF provides a measure of particle sizes in the selected measurement sites (volume $=50 \mu \mathrm{m}^{3}$ ). Studies $^{31}$ in the lens found that DLS was significantly more sensitive than the Scheimpflug photography in detecting early changes in the lens. When the DLS probe was used to obtain measurements from the entire vitreous body, scanning was performed in conjunction with a micropositioning assembly, which controlled detector position in the $x, y$, and $z$ planes. This enabled semi-automated measurements from a sufficient number of sites within the bovine vitreous body to create a three-dimensional map of the distribution of the average particle sizes of vitreous macromolecules (Figure 13). Furthermore, in studies ${ }^{32}$ of autopsy human eyes, DLS was able to detect the structural changes ${ }^{16}$ resulting from Diabetic Vitreopathy. ${ }^{25}$

\section{Conclusions}

No single method presently exists that will enable accurate and reproducible non-invasive imaging of both the vitreous body and the vitreo-retinal interface. This impacts significantly upon the ability to assess the effects of aging and disease and, in particular, upon the accuracy of diagnosing posterior vitreous detachment clinically. Moreover, this limitation hinders our ability to adequately evaluate the role of vitreous in vitreo-retinal diseases such as retinal detachment, both in general terms and in specific clinical cases.

Today, combining more than one of the aforementioned techniques could provide considerably more information than just one technique. For example, NMR Spectroscopy could assess the degree of vitreous liquefaction, Dynamic Light Scattering could determine the concurrent aggregation of collagen and other macromolecules that occurs during liquefaction, Raman Spectroscopy could identify the presence of specific molecular moieties that provide insight into pathogenesis, while Optical Coherence Tomography could image the vitreo-retinal interface. Hopefully, the future will witness the combination of these and other techniques into a single non-invasive instrument for research and clinical applications.

\section{References}

1 Duke-Elder SW. The nature of the vitreous body. Brit J Ophthalmol (suppl IV) 1930; 14: 6.

2 Redslob E. Le Corps Vitre. Societe Francaise d'Ophtalmologie Monogr. Masson: Paris, 1932, pp 174178

3 Eisner G. Biomicroscopy of the Peripheral Fundus. SpringerVerlag: New York, 1973.

4 Worst JGF. Cisternal systems of the fully developed vitreous body in the young adult. Trans Ophthalmol Soc UK 1977; 97: 550-554.

5 Sebag J, Balazs EA. Morphology and ultrastructure of human vitreous fibers. Invest Ophthalmol Vis Sci 1989; 30: 1867-1871.

6 Kishi S, Shimizu K. Posterior precortical vitreous pocket. Arch Ophthalmol 1990; 108: 979.

7 Sebag J. Letter to the Editor. Arch Ophthalmol 1991; 190: 1059.

8 Foulds WS. Is your vitreous really necessary? The role of the vitreous in the eye with particular reference to retinal attachment, detachment and the mode of action of vitreous substitutes. (The 2nd Duke-Elder Lecture). Eye 1987; 1: 641-664.

9 Sebag J. The Vitreous: Structure, Function and Pathobiology. Springer-Verlag: New York, 1989.

10 Sebag J. Macromolecular structure of vitreous. Prog Polym Sci 1998; 23: 415-446.

11 Faulborn J, Bowald S. Microproliferations in proliferative diabetic retinopathy and their relation to the vitreous. Graef Arch Clin Exp Ophthalmol 1985; 223: 130.

12 Dunker S, Glinz J, Faulborn J. Morphologic studies of the peripheral vitreoretinal interface in humans reveal structures implicated in the pathogenesis of retinal tears. Retina 1997; 17: 124-130.

13 Sebag J, Balazs EA. Human vitreous fibres and vitreoretinal disease. Trans Ophthalm Soc UK 1985; 104: 123.

14 Sebag J. Age-related changes in human vitreous structure. Graef Arch Clin Exp Ophthalmol 1987; 225: 89-93.

15 Sebag J. Age-related differences in the human vitreoretinal interface. Arch Ophthalmol 1991; 109: 966-971.

16 Sebag J. Abnormalities of human vitreous structure in diabetes. Graef Arch Clin Exp Ophthalmol 1993; 231: 257260

17 Sebag J. Classifying posterior vitreous detachment-a new way to look at the invisible. Brit J Ophthalmol 1997; 81: 521-522.

18 Schepens CI, Trempe CL, Takahashi M. Atlas of Vitreous Biomicroscopy. Butterworth Heinemann: Boston, 1999.

19 Mainster MA, Timberlake GT, Webb RH, Hughes GW Scanning laser ophthalmoscopy—clinical applications. Ophthalmology 1982; 89: 852-857.

20 Oksala A. Ultrasonic findings in the vitreous body at various ages. Alb v. Graef Arch Klin Exp Ophthalmol 1978; 207: 275-280.

21 Chu T, Lopez PF, Cano MR, Green RL. Posterior vitreoschisis - an echographic finding in proliferative diabetic retinopathy. Ophthalmology 1996; 103: 315-322.

22 Sebag J, Buckingham B, Charles MA, Reiser K. Biochemical abnormalities in vitreous of humans with proliferative diabetic retinopathy. Arch Ophthalmol 1992; 110: $1472-1479$.

23 Fujimoto JG, Brezinski ME, Tearney GJ et al. Optical 
biopsy and imaging using optical coherence tomography. Nature Med 1995; 1: 970-972.

24 Gaudric A, Haouchine B, Massin P et al. Macular hole formation-new data provided by optical coherence tomography. Arch Ophthalmol 1999; 117: 744-751.

25 Sebag J. Diabetic vitreopathy (Guest Editorial). Ophthalmology 1996; 103: 205-206.

26 Aguayo J, Glaser B, Mildvan A et al. Study of vitreous liquifaction by NMR spectroscopy and imaging. Invest Ophthalmol Vis Sci 1985; 26: 692-697.

27 Sebag J. Pharmacologic vitreolysis (Guest Editorial). Retina 1998; 18: 1-3.

28 Nie S, Bergbauer KL, Kuck JFR Jr, Yu NT. Near infrared fourier transform raman spectroscopy in human lens research. Exp Eye Res 1990; 51: 619-623.
29 Sebag J, Nie S, Reiser KA, Charles MA, Yu NT. Raman spectroscopy of human vitreous in proliferative diabetic retinopathy. Invest Ophthalmol Vis Sci 1994; 35: 2976-2980.

30 Ansari RR, Dunker S, Suh K, Kitaya N, Sebag J.

Quantitative molecular characterization of bovine vitreous and lens with non-invasive dynamic light scattering. Exp Eye Res 2001; 73: 859-866.

31 Ansari RR, Datiles MB III, King JF, Leftwood D. Measuring lens opacity: combining quasi-elastic light scattering with Scheimpflug imaging system. SPIE 1998; 3246: 35-43.

32 Sebag J, Ansari RR, Dunker S, Suh SI. Dyanmic light scattering of diabetic vitreopathy. Diabetes Technology $\mathcal{E}$ Therapeutics 1999; 1: 169-176. 